\section{Human Y5 RNA specializes a Ro ribonucleoprotein for $5 \mathrm{~S}$ ribosomal RNA quality control}

\author{
J. Robert Hogg ${ }^{1,3}$ and Kathleen Collins ${ }^{2}$ \\ Department of Molecular and Cell Biology, University of \\ California at Berkely, Berkeley, California 94720 USA
}

Humans express four distinct non-protein-coding $Y$ RNAs (ncRNAs). To investigate Y RNA functional diversification, we exploited an RNA-based affinity purification method to isolate ribonucleoproteins (RNPs) assembled on individual human Y RNAs. Silver staining and mass spectrometry revealed that the Ro and La proteins assemble with all Y RNAs, while additional proteins associate with specific Y RNAs. Unexpectedly, Y5 RNA uniquely copurified ribosomal protein L5 and its binding partner 5S RNA. These findings reveal a contribution of $\mathrm{Y} 5$ to $5 \mathrm{~S}$ surveillance and suggest that interactions between Ro-Y5 and L5-5S RNPs establish 5S RNA as a target of quality control.

Supplemental material is available at http://www.genesdev.org.

Received August 13, 2007; revised version accepted October 11, 2007.

Empirical and computational approaches have revealed a dramatically expanded complexity of non-protein-coding RNA (ncRNA) in higher eukaryotes (Taft et al. 2007). For example, while nonvertebrate metazoans such as Caenorhabditis elegans and a few microbes including the eubacterium Deinococcus radiodurans have a single Y RNA, vertebrates encode up to four Y RNAs (Chen and Wolin 2004; Perreault et al. 2007). Y RNAs are pyrimidine-rich transcripts of $\sim 100$ nucleotides (nt) synthesized by RNA Polymerase III that possess a sequence-specific binding site for the Ro protein formed by base-pairing between the $5^{\prime}$ and $3^{\prime}$ ends of the Y RNA transcript. Evidence from multiple organisms has demonstrated that Ro association with this perfectly conserved motif is required for Y RNA accumulation in vivo (Chen and Wolin 2004). Biochemical, genetic, and structural studies implicate Ro proteins in pathways of ncRNA quality control and ribosomal RNA processing (Labbe et al. 1999; Chen and Wolin 2004; Stein et al. 2005; Fuchs et al. 2006; Chen et al. 2007). The function of Y RNA in Ro ribonucleoproteins (RNPs) has remained elusive, but a model has been suggested in which Y RNAs inhibit Ro function by steric occlusion of the Ro-binding surface for misfolded or misprocessed ncRNA (Stein et al. 2005).

[Keywords: RNA Affinity in Tandem (RAT); noncoding RNA; quality control surveillance]

${ }^{1}$ Present address: Howard Hughes Medical Institute and Department of Biochemistry and Molecular Biophysics, Columbia University, New York, NY 10032, USA.

Correspondence.

${ }^{2}$ E-MAIL kcollins@berkeley.edu; FAX (510) 643-6334.

${ }^{3}$ E-MAIL jh2721@columbia.edu; FAX .

Article is online at http://www.genesdev.org/cgi/doi/10.1101/gad.1603907.
This role could account for Y RNA function in organisms with a single Y RNA (Chen et al. 2007), but it does not provide a rationale for the observed sequence conservation of multiple distinct $\mathrm{Y}$ RNAs in vertebrates (Perreault et al. 2007).

Here we show that individual human Y RNAs have unique protein and ncRNA interaction specificities. We demonstrate that all four human Y RNAs (Y1, Y3, Y4, and Y5) assemble with the core Y RNP proteins Ro and $\mathrm{La}$, and additional proteins exhibit specific interactions with subsets of Y RNPs. Of the human Y RNAs, only Y5 copurifies ribosomal protein L5 and an extensively characterized target of Ro-mediated quality control, 5S ribosomal RNA. We propose that Y RNAs play both positive and negative roles in establishing the target specificity of ncRNA surveillance by Ro. Our findings provide new insights about the functions of ncRNA and the significance of expansion of vertebrate ncRNA diversity.

\section{Results and Discussion}

RNA-based affinity purification of individual Y RNPS

An RNA-based method of affinity purification was required to separate the RNPs endogenously assembled on human Y1, Y3, Y4, and Y5 RNAs. For this purpose, we developed an RNP purification method termed RNA Affinity in Tandem (RAT). Each Y RNA was expressed in vivo as a fusion to an affinity tag composed of two hairpins: one binding the Pseudomonas phage 7 (PP7) coat protein $(\mathrm{CP})$ and one binding the antibiotic tobramycin (Hogg and Collins 2007). Each Y RNA was tagged at the $5^{\prime}$ or $3^{\prime}$ end with one or both orientations of the tag hairpins and expressed under the control of the commonly exploited human U6 RNA Polymerase III promoter by transient transfection of human $293 \mathrm{~T}$ cells. The most uniform Y RNA expression levels were obtained using a 3' tag with the PP7 hairpin followed by the tobramycin hairpin (data not shown). Cells expressing the entire panel of Y RNAs with this tag configuration and a parallel empty vector control were lysed to generate whole-cell extracts. Recombinant PP7CP tagged with the two Protein A domains (ZZ) and Tobacco Etch Virus (TEV) protease cleavage site of the protein tandem affinity purification (TAP) tag (Puig et al. 2001) was added to cell extract, and complexes harboring tagged RNA were recovered on IgG agarose. Following elution by TEV protease, tagged RNPs were bound to tobramycin resin and eluted by denaturation.

RAT-purified samples were split for detection of RNA and protein. RNA was extracted from tobramycin resin, resolved by PAGE, and detected by direct staining with SYBR Gold. Each tagged Y RNA was purified to near homogeneity, with no endogenous Y RNA copurification (the migration of each tagged Y RNA as a doublet was confirmed by Northern blot hybridization) (Fig. 1A). Associated proteins were detected by silver staining after SDS-PAGE. Three prominent polypeptides were present in all tagged Y RNA samples but absent from the mock affinity purification (Fig. 1B). The smallest of these is CP recovered on tobramycin resin through its association with the PP7 hairpin of the RAT tag. The additional polypeptides correspond to the La and Ro autoantigens, as confirmed by immunoblots (data not shown) and mass 




Figure 1. Purification of RAT-tagged human Y RNPs. Extracts from cells expressing RAT-tagged Y RNAs or empty vector were used for affinity purification. (A) RNA from whole-cell extract (WCE) or tobramycin resin was fractionated by denaturing PAGE and visualized by SYBR Gold staining. (B) Proteins bound to tobramycin resin were resolved by SDS-PAGE and visualized by silver staining. The mobilities of Ro, La, and CP are indicated. Polypeptides of $\sim 30 \mathrm{kDa}$ and $\sim 45 \mathrm{kDa}$ evident in both purifications derive from nonspecific resin binding of a small fraction of the vast excess of TEV protease used to elute RNPs in the first step of affinity purification.

spectrometry (see below). Antibodies against both of these proteins were previously shown to immunoprecipitate Y RNAs (Chen and Wolin 2004), validating the use of RAT for affinity purification of physiologically relevant RNPs. The 3' tag placement could have influenced the stoichiometry of La association, but we note that 5'-tagged Y RNAs also copurified both Ro and La (data not shown). We conclude from these and previous findings that all of the human Y RNAs share their major protein interaction partners, Ro and La.

This result suggests that any specialized Ro RNP function conferred by a particular Y RNA is likely to involve transient or substoichiometric associations with other factors. Proteins suggested to interact with Y RNPs include nucleolin and hnRNP I/PTB, both of which are reported to bind only Y1 and Y3 (Fabini et al. 2000; Fouraux et al. 2002), and Ro RNP-Binding Protein I (RoBPI/FIR/PUF60), a protein identified from diverse assays, including a yeast three-hybrid screen with the combination of Y5 RNA and Ro as bait (Page-McCaw et al. 1999; Bouffard et al. 2000; Liu et al. 2000). To investigate the existence and specificity of substoichiometric interaction partners of an individual human Y RNA, we selected Y5 RNA for additional study. Y5-RAT RNA recapitulated the higher abundance and preferential nuclear localization of endogenous Y5 RNA (data not shown) that distinguishes Y5 among the human Y RNAs (Gendron et al. 2001). In addition, the positive selection of Y5 RNA in primate evolution is indicative of functional specialization (Maraia et al. 1996).

We performed large-scale affinity purifications from parallel Y5-RAT and control cell extracts to identify proteins bound to Y5 RNA by mass spectrometry. A small aliquot of sample eluted from tobramycin resin was assessed by SDS-PAGE and silver staining (Supplementary
Fig. 1A), and the majority of the sample was processed for protein identification (Supplementary Fig. 1B). The Y5-RAT affinity purification specifically enriched for exogenous CP and endogenous La and Ro as expected, with 30 and 28 unique peptides identified from Ro and La, respectively. Two additional proteins specific to the Y5RAT sample were represented by five unique peptides each. One of these was RoBPI, isolated previously as an interaction partner of Y5 RNA and Ro (Bouffard et al. 2000). The second was interferon-induced protein with tetratricopeptide repeats 5 (IFIT5), a protein of unknown function. One additional protein, ribosomal protein L5, was identified with a relatively high coverage of four unique peptides (Supplementary Fig. 1B). As described below, we first characterized RoBPI and IFIT5 followed by L5.

\section{RNA-binding specificity of RoBPI and IFIT5}

To confirm the association of RoBPI and IFIT5 with Y5 RNA, we expressed tagged RoBPI and IFIT5 and assayed their ability to copurify endogenous Ro and Y RNA. Flagtagged proteins were expressed by transient transfection of $293 \mathrm{~T}$ cells, and complexes harboring tagged RoBPI or IFIT5 were affinity-purified from whole-cell extract by binding to antibody resin. By immunoblot, Ro antibody recognized a protein of the expected size in input extracts and in the complexes of purified RoBPI and IFIT5 (Fig. 2A, lanes 1-4,6). Other Flag-tagged proteins did not copurify a detectable level of Ro (see Fig. 2A, lanes 8,9; data not shown). Importantly, pretreatment of cell extracts with RNase A eliminated the copurification of Ro with RoBPI and IFIT5 (Fig. 2A, lanes 5,7). This finding suggests that Ro is associated with RoBPI and IFIT5 in a manner dependent on Y RNA and/or other RNA(s). We examined the RNA interaction specificities of RoBPI and IFIT5 by direct staining of RNAs that copurified with the tagged proteins. RoBPI and IFIT5 both enriched Y5 and other RNAs (Supplementary Fig. 2A, left panel). The previously uncharacterized IFIT5 protein copurified a diverse mixture of small RNAs, predominantly $<150 \mathrm{nt}$ in length. Northern blot hybridization assays revealed that Y5 was a minor component of the overall RNA population bound to IFIT5 (Supplementary Fig. 2A, right panel), and thus, this protein is not further described here (see Supplementary Fig. 2B,C and the legend for additional studies of IFIT5-associated RNAs and proteins).

RoBPI has been suggested to bind Y5 but not Y1, Y3, or Y4 RNP based on assays in yeast and human cell extract (Bouffard et al. 2000). In order to establish the Y RNAbinding specificity of RoBPI in vivo, we used formaldehyde cross-linking prior to cell lysis to trap endogenous interactions. Cross-linking followed by affinity purification under stringent denaturing conditions removes the caveats of potential post-lysis mixing or variable subunit dissociation that complicate analysis of interaction assays using native cell extract (Niranjanakumari et al. 2002). TAP-tagged RoBPI was expressed using the retroviral long-terminal repeat promoter of the $\mathrm{pBABE}$ vector in 293 T cells, which we find gives near-endogenous expression levels for most proteins (Hogg and Collins 2007). RNA was isolated from cross-linked input extracts and IgG-bound samples, and cross-links were reversed prior to Northern blot analysis. Under these assay conditions, tagged RoBPI enriched Y5 RNA and also Y1 
A

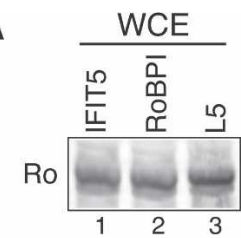

B
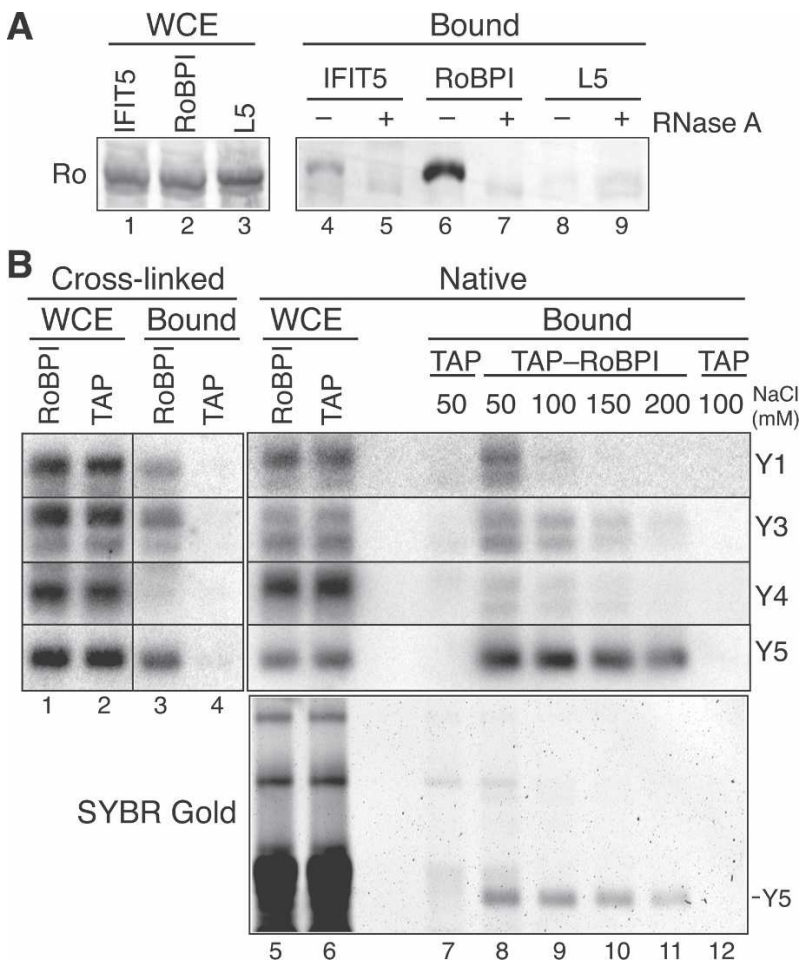

Native

Figure 2. Interaction of RoBPI and IFIT5 with Y RNPs. $(A)$ The indicated Flag-tagged proteins were purified from whole-cell extract (WCE) that had been preincubated with or without RNase A. Endogenous Ro was detected by immunoblot. $(B)$ WCE harboring TAPRoBPI or the TAP tag alone was prepared from cells cross-linked with formaldehyde (lanes 1,2) or under native conditions (lanes 5,6). Affinity purification was performed under stringent denaturing conditions (lanes 3,4) or native conditions with extract adjusted to the indicated $\mathrm{NaCl}$ concentrations (lanes 7-12). Individual Y RNAs were detected by Northern blot; lanes $1-4$ or 5-12 were from the same exposure of the same membrane. (Bottom panel, lanes 5-12) RNAs in WCE and bound fractions were resolved by denaturing PAGE and detected by SYBR Gold staining.

and Y3, with little or no specific enrichment of Y4 (Fig. $2 \mathrm{~B}$, lanes 1,3$)$. As a control, affinity purification using extract from cells expressing the TAP tag alone did not enrich any of the Y RNAs (Fig. 2B, lanes 2,4).

For comparison with previous results, we examined RoBPI copurification of Y RNAs using native cell extract. Prior to affinity purification, aliquots of extract were adjusted to varying ionic strength. SYBR Gold staining and Northern blot hybridization of RNAs enriched with TAP-tagged RoBPI (Fig. 2B, lanes 8-11) or the control TAP tag alone (Fig. 2B, lanes 7,12 ) revealed that $\mathrm{Y} 5$ is the predominant ncRNA interaction partner of RoBPI. In extract containing $50 \mathrm{mM} \mathrm{NaCl}$ (Fig. 2B, lane 8), RoBPI copurified Y5, Y1, and Y3 RNAs but copurified little if any Y4 RNA. This parallels the profile observed when in vivo interactions were trapped by cross-linking (Fig. 2B, lane 3). In contrast, in extract adjusted to 150$200 \mathrm{mM} \mathrm{NaCl}$ (Fig. 2B, lanes 10,11), only Y5 remained substantially associated with RoBPI. In sum, our results indicate that RoBPI associates with Y5 as described previously (Bouffard et al. 2000) and also binds Y1 and Y3 RNPs. We conclude that RoBPI is not a unique binding partner of Y5 but does engage in specific interactions with a subset of Y RNAs.
Y5 RNP association with ribosomal protein L5 and variant $5 S$ RNA

Mass spectrometry of Y5 RNPs isolated by RAT identified ribosomal protein L5 with a coverage of four unique peptides versus a single peptide identified in the mock purification (Supplementary Fig. 1B). Independently, we identified L5 in an excised gel slice containing a protein specifically coenriched by Y5-RAT (data not shown). These identifications implicate L5 as a potential binding partner of Y5 RNA. We investigated the putative association of L5 protein and Y5 RNA using the approaches described above for RoBPI. By transient transfection of $293 \mathrm{~T}$ cells with pBABE vectors, we expressed TAPtagged L5 and the negative controls TAP-tagged ribosomal protein L9 (similar to L5 in size and isoelectric point) and the TAP tag alone. Cells were either crosslinked with formaldehyde prior to cell lysis for affinity purification under stringent denaturing conditions (Supplementary Fig. 3A) or lysed in low ionic strength buffer for affinity purification under native conditions (Supplementary Fig. 3B). Under both conditions, TAP-L5 specifically copurified a small amount of endogenous Y5 RNA. A low efficiency of endogenous Y5 recovery was expected, because transiently expressed TAP-L5 must compete for interactions made by the highly abundant endogenous L5. Although TAP-L5 copurified a relatively small quantity of Y5 RNA, this copurification was highly specific. No other Y RNAs were copurified with TAP-L5, and TAP-L9 or the TAP tag alone did not copurify any of the human Y RNAs (Supplementary Fig. 3A,B).

L5 is the binding partner of 5S RNA, an independently transcribed ribosomal RNA synthesized by RNA Polymerase III. L5 packages 5S RNA for stability and transport prior to L5-5S RNP incorporation into larger ribosome particles (Steitz et al. 1988). Studies of Xenopus Ro binding to variant 5S RNA provide the major supporting evidence for a model of Ro function in which free Ro mediates quality control of misfolded or misprocessed ncRNA (O'Brien and Wolin 1994; Shi et al. 1996; Green et al. 1998; Stein et al. 2005; Fuchs et al. 2006). The copurification of L5 with Y5 RNA described above raised an intriguing possibility: 5S RNA recognition could instead occur in the context of Y5-Ro RNP association with 5S-L5 RNP (an RNP-RNP rather than proteinRNA recognition model for quality control surveillance). We were unable to conclusively establish that the Y5 RNA-L5 protein complex also contains Ro, due to the technical limitation of Flag-tagged L5 competition with endogenous L5 for copurification of Ro (Fig. 2A, lanes 8, 9). However, the presence of Ro in this Y5 complex can be inferred with high likelihood from the fact that Y RNA stability is exquisitely dependent on Ro in vivo.

The RNP-RNP model for quality control surveillance requires that the Y5-L5 complex contain variant (or wild-type misfolded) 5S RNA. Consistent with this model, C. elegans lacking Ro show increased incorporation of variant 5S RNA into ribosomes (Labbe et al. 1999), indicating that the variant 5S RNAs discriminated by Ro can assemble with L5. To examine the proposed RNP-RNP interaction hypothesis, we assayed for the ability of tagged Y5 RNA to copurify variant 5S RNA. Because endogenous human 5S RNA undergoing Romediated quality control would be expected to be scarce, we coexpressed each Y-RAT RNA or an empty vector con- 
trol with a variant human 5S RNA modeled on a biochemically characterized Xenopus pre-5S RNA known to misfold and be recognized by Ro (Shi et al. 1996). The human 5S RNA variant has a uridine-rich singlestranded 3 ' extension that is particularly important for recognition by Ro (Supplementary Fig. 4), which gave the variant RNA an electrophoretic mobility readily distinguishable from that of the wild-type 5S RNA.

Whole-cell extracts were used for affinity purification through the first step of RAT. RNAs from the input and eluate fractions of Y-RAT and mock-affinity purifications were probed by Northern blot using oligonucleotides complementary to the RAT tag and to a sequence shared in the variant and wild-type 5S RNAs. Each of the RAT-tagged Y RNAs was robustly expressed (Fig. 3A, bottom panel, lanes 1-5) and enriched following affinity purification (Fig. 3A, lanes 6-10). Of the four human Y RNAs, only Y5-RAT copurified 5S RNA above the low background detected in the mock affinity purification (Fig. 3A, top panel, lanes 6-10). Variant and endogenous 5S RNAs were both recovered in association with Y5RAT, but the variant 5S RNA was strikingly enriched.

A


B

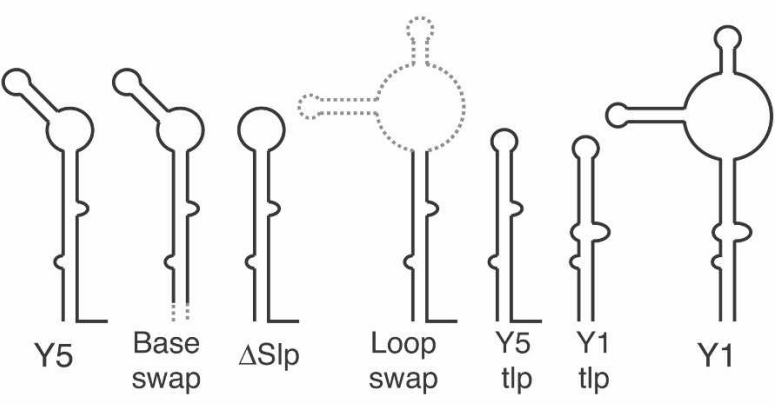

C

WCE

Bound

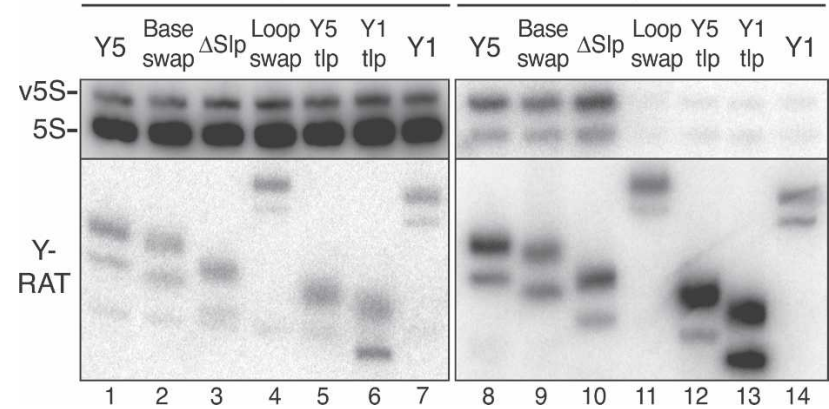

Figure 3. Y RNA sequence requirements for interaction with variant 5S RNA. $(A, C)$ Variant 5S RNA (v5S) was coexpressed with RAT-tagged Y RNA, chimeric or mutant Y RNA illustrated in $B$, or empty vector. RNPs assembled on RAT-tagged RNAs were recovered from whole-cell extract (WCE) by binding to IgG agarose. RNA was extracted from WCE or bound fractions and resolved by denaturing PAGE for Northern blots.
This specificity was not a trivial consequence of variant 5S RNA coexpression with Y5-RAT, because coexpressed wild-type 5S RNA was not similarly enriched (data not shown). We repeated these studies using extracts from formaldehyde cross-linked cells with similar results (data not shown). We conclude that human Y RNPs harboring Y5 associate with 5S RNA in a manner that is highly preferential for a misfolded $5 \mathrm{~S}$ variant.

\section{Y RNA sequence requirements for $5 S$ RNA association}

Compared with the other human Y RNAs, Y5 has a longer 3' extension and a smaller pyrimidine-rich internal loop. To examine the contribution of Y5 RNA sequence to 5S RNA recognition, we assayed variant $5 \mathrm{~S}$ RNA copurification with a panel of RAT-tagged chimeric and mutant Y RNAs (Fig. 3B; Supplementary Table 1), all of which retained the stem region essential for Ro binding. The chimeric and mutant Y-RAT RNAs were all robustly expressed (Fig. 3C, bottom panel, input lanes 1-7), comparably partitioned into both the nucleus and cytoplasm based on subcellular fractionation (data not shown), and efficiently purified under native conditions (Fig. 3C, bottom panel, lanes 8-14). The positive control Y5-RAT copurified variant 5S RNA and some endogenous 5S RNA, while the negative control Y1-RAT did not (Fig. 3C, lanes 8,14). Replacing Y5 nucleotides flanking the Ro-binding stem with the corresponding portion of Y1 RNA (base swap) (Fig. 3C, lane 9) or deleting the internal Y5 stem-loop formed by nucleotides $27-$ $42(\Delta S l p)$ (Fig. 3C, lane 10$)$ did not reduce association with 5S RNA. In contrast, replacing the entire internal loop region of Y5 (residues 22-52) with the analogous loop region of Y1 (residues 22-86) abrogated Y RNP copurification of 5S RNA (loop swap) (Fig. 3C, lane 11).

These observations suggest two possible scenarios. The Y5 loop region could contain sequences that promote interaction with 5S RNA, or the larger loop of Y1 could sterically inhibit 5S RNA binding to Ro. To evaluate these models, we assayed 5S RNA copurification with mutant Y RNAs in which the entire Y1 or Y5 internal loop region was replaced by a UUCG tetraloop (Fig. 3B, tlp substitutions). These internal deletions did not reduce Y RNA accumulation or stability in extract (Fig. 3C, lanes 5,6). However, neither Y1 tlp nor Y5 tlp copurified 5S RNA (Fig. 3C, lanes 12,13). Together, the results above suggest that particular features of the Y5 internal loop confer to Y5 RNP the ability to recognize variant 5S RNA in RNP. The Y5 internal loop has also been proposed to determine the specificity of RoBPI interaction (Bouffard et al. 2000). We were unable to detect copurification of variant 5S RNA with RoBPI (data not shown), consistent with a mutually exclusive interaction of Y5 RNP with either RoBPI or 5S RNP.

\section{Ro sequence requirements for Y5 and 5S RNA interaction in vivo}

In vitro assays using purified Ro suggest that Y RNA and misfolded 5S RNA compete for Ro binding. If the physiological surveillance of misfolded $5 \mathrm{~S}$ is performed by Ro RNP rather than free Ro and L5-5S RNP rather than 5S RNA alone is the target of surveillance, then the Ro requirements for $5 \mathrm{~S}$ recognition in vivo may differ from the requirements established previously in vitro. To investigate Ro structural requirements for variant 5S RNA 
association, we used transient transfection of $293 \mathrm{~T}$ cells to express mutant Ro proteins with amino acid substitutions that alter two surfaces of the protein (Stein et al. 2005; Fuchs et al. 2006): an extended surface important for Ro binding to Y RNA and misfolded RNA (R184A and $\mathrm{H} 187 \mathrm{~S}$ ), and a central cavity important for Ro binding to misfolded RNA (R174A and the double-substitution K170A/R174A) in vitro. We coexpressed TAPtagged wild-type or mutant Ro protein with variant $5 \mathrm{~S}$ RNA. Ro with the H187S substitution accumulated to very low levels in vivo (Fig. 4A), while the remaining proteins were expressed at levels similar to TAP-tagged wild-type Ro (Fig. 4A) and untagged endogenous Ro (data not shown).

We first performed affinity purifications using extracts from cells treated with formaldehyde to trap in vivo interactions. Wild-type Ro copurified endogenous Y5 RNA and variant 5S RNA as expected, while the TAP tag alone did not (Fig. 4B, lanes 1,6). When normalized by protein expression level (Fig. 4A), all of the mutant Ro proteins copurified Y5 RNA with an efficiency similar to wild-type Ro (Fig. 4B, lanes 2,4,5). Curiously, all of the mutant Ro proteins also copurified variant 5S RNA as efficiently as wild-type Ro (Fig. 4B, lanes 2,4,5). To reconcile these results with previous results from in vitro binding assays using purified Ro, we repeated the affinity purification of TAP-tagged wild-type and mutant Ro proteins using extracts from cells lysed by low ionic strength without prior cross-linking. Under native conditions that allow subunit dissociation of endogenously assembled complexes, the mutant Ro proteins showed moderate (R184A) or severe (R174A, K170A/R174A) defects in binding to variant 5S RNA (Fig. 4B, lanes 8,10,11) compared with wild-type Ro (Fig. 4B, lane 7). Severe de-
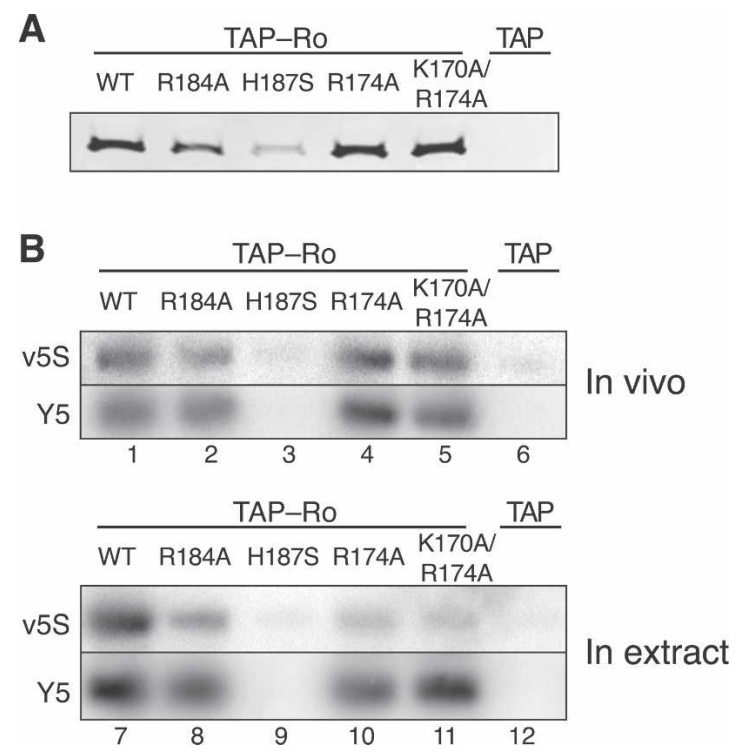

Figure 4. Ro sequence requirements for binding Y5 RNA and variant 5S RNA. Cells were transfected to express variant 5S RNA (v5S) and TAP-tagged wild-type (WT) Ro, mutant Ro, or the TAP tag alone. (A) Whole-cell extracts (WCE) made under native conditions were used to detect tagged Ro by immunoblot with rabbit IgG. $(B)$ TAP-tagged proteins were affinity-purified under stringent denaturing conditions from cells cross-linked with formaldehyde (lanes 1-6) or under native conditions (lanes 7-12). RNA was extracted from WCE or bound fractions and resolved by denaturing PAGE for Northern blots. fects were observed for Ro mutants with substitutions in basic residues of the central cavity proposed to engage a ncRNA 3' end. We suggest that the mutant Ro proteins previously shown to be impaired for binding to misfolded RNA in vitro retain an ability to surveil and bind 5S RNP in their Ro RNP context, but the Ro-Y5 RNPs harboring mutant Ro proteins dissociate from 5S RNP more readily in cell extract. In sum, these results demonstrate that the sequence requirements for Ro association with a ncRNA quality control substrate in vitro did not predict the sequence requirements for Ro association with ncRNA in vivo, consistent with a role for additional factors such as Y RNA and L5 in the in vivo process of quality control surveillance.

\section{Implications for quality control surveillance}

Our findings suggest that ncRNA quality control surveillance by Ro involves discrimination of variant 5S RNA in the context of an association between L5 RNP and Y5 RNP. In this RNP-RNP recognition model, a large network of protein-RNA, protein-protein, and perhaps RNA-RNA interactions could increase the efficiency and specificity of ncRNA target discrimination. This network may be particularly important for surveillance of ncRNA quality control targets of low abundance (such as variant 5S RNA in human cells) but may be dispensable for recognition of variant 5S RNA stockpiled in Xenopus oocytes. We show here that the internal loop of human Y5 RNA harbors specificity elements for association with 5S RNP. This internal loop region differs in sequence among the human Y RNAs, implying that each Y RNA could enhance Ro recognition of a distinct target. Y RNPs can be envisioned as a modular system for misfolded RNA recognition, in which Y RNAs and Ro each possess conserved surfaces required for core Y RNP assembly and distinct surfaces adapted to recognize quality control targets. For Y RNAs, conservation of the Robinding site and divergence of other structural features would allow the diversification of vertebrate Y RNA function in parallel with the evolutionary emergence of new ncRNA variants in need of quality control.

In retrospect, L5 is an excellent candidate for involvement in 5S RNA quality control, because the intracellular trafficking of $5 \mathrm{~S}$ prior to ribosome incorporation occurs in the context of an L5-5S RNP (Steitz et al. 1988). In one possible model to account for the role of L5 in 5S surveillance, an RNA-binding surface of L5 unoccupied in the ribosome-free 5S RNP could bind to Y5 RNA such that the 3' end of 5S RNA is positioned above the central cavity of Ro. This model allows Y5 RNA to have both positive and negative influences on Ro-mediated ncRNA surveillance. Y5 can stimulate Ro association with 5S RNP and potentially other quality control targets (the positive role) while acting as a gatekeeper for the surface of Ro that discriminates folded from misfolded ncRNA (the negative role). It is important to note that our experiments do not address what happens to a Y RNA following Ro RNP recognition of a ncRNA quality control substrate. Ro RNP remodeling after commitment to ncRNA target turnover could release the Y RNA, or the Y RNA could play an additional role in recruiting nucleases such as the activity associated with IFIT5 (Supplementary Fig. 2). The scarce nature of quality control intermediates has prevented progress in understanding the enzymatic machinery responsible for their turnover. The 
studies presented here will facilitate future investigations of the mechanisms used by quality control surveillance complexes to direct subsequent ncRNA degradation.

\section{Materials and methods}

\section{Constructs}

Human Y RNA sequences were cloned in fusion with RAT-tag cassettes (Hogg and Collins 2007) in pBluescript immediately downstream from a 284-base-pair (bp) region of the human U6 RNA Polymerase III promoter. The variant human 5S RNA was designed based on the Xenopus pre-5S stem $\mathrm{mt} 1+2$ (Shi et al. 1996) and was expressed using $227 \mathrm{bp}$ of upstream genomic sequence cloned into pBluescript. Human Ro, RoBPI, L5, and L9 ORFs were fused to the N-terminal TAP tag in pBABE or to an N-terminal triple Flag tag in pcDNA 3.1 as indicated. The IFIT5 ORF was fused to an N-terminal triple Flag tag in pcDNA 3.1.

Cell culture and extracts

Phoenix 293T cells were maintained in DMEM supplemented with $10 \%$ fetal calf serum and antibiotics. Cells were transfected using calcium phosphate, and whole-cell extracts were prepared by hypotonic freezethaw lysis. Y-RAT purifications shown in Figure 1 and Supplementary Figure 1 were performed at $150 \mathrm{mM} \mathrm{NaCl}$; other purifications were performed at $100 \mathrm{mM} \mathrm{NaCl}$ unless indicated. For cross-linking, cells were treated with $0.1 \%$ formaldehyde for $10 \mathrm{~min}$ at room temperature prior to lysis and extract sonication (Hogg and Collins 2007). Nuclease treatment of cell extract was performed for $2 \mathrm{~h}$ at $4^{\circ} \mathrm{C}$ in the presence or absence of $500 \mu \mathrm{g} / \mathrm{mL}$ RNase A.

\section{Affinity purifications and mass spectrometry}

Purification of PP7CP, preparation of tobramycin resin, RAT purification, protein-based affinity purification, and mass spectrometry were performed as described (Hogg and Collins 2007), with minor modifications. For mass spectrometry, RNP bound to tobramycin resin was eluted in 2.5 packed gel volumes of $100 \mathrm{mM}$ ammonium carbonate $(\mathrm{pH} 10)$ and dried by vacuum. TAP-tagged proteins were bound to IgG resin. Flag-tagged proteins were bound to EZview Red Anti-Flag M2 Affinity Gel (Sigma). RNA was stained with SYBR Gold (Invitrogen), and Northern blots were hybridized with end-labeled oligonucleotides. Ro protein was detected using a rabbit polyclonal antibody (H-300; Santa Cruz Biotechnology).

\section{Acknowledgments}

We thank Don Rio, Marco Blanchette, and Lori Kohlstaedt for assistance with mass spectrometry, and Dragony Fu for contributing protein expression constructs. Funding was provided by the University of California Cancer Research Coordinating Committee.

\section{References}

Bouffard, P., Barbar, E., Briere, F., and Boire, G. 2000. Interaction cloning and characterization of RoBPI, a novel protein binding to human Ro ribonucleoproteins. RNA 6: 66-78.

Chen, X. and Wolin, S.L. 2004. The Ro $60 \mathrm{kDa}$ autoantigen: Insights into cellular function and role in autoimmunity. J. Mol. Med. 82: 232-239.

Chen, X., Wurtmann, E.J., Van Batavia, J., Zybailov, B., Washburn, M.P., and Wolin, S.L. 2007. An ortholog of the Ro autoantigen functions in 23S rRNA maturation in D. radiodurans. Genes \& Dev. 21: 13281339.

Fabini, G., Rutjes, S.A., Zimmermann, C., Pruijn, G.J., and Steiner, G. 2000. Analysis of the molecular composition of Ro ribonucleoprotein complexes. Identification of novel Y RNA-binding proteins. Eur. I. Biochem. 267: 2778-2789.

Fouraux, M.A., Bouvet, P., Verkaart, S., van Venrooij, W.J., and Pruijn, G.J. 2002. Nucleolin associates with a subset of the human Ro ribonucleoprotein complexes. J. Mol. Biol. 320: 475-488.

Fuchs, G., Stein, A.J., Fu, C., Reinisch, K.M., and Wolin, S.L. 2006. Structural and biochemical basis for misfolded RNA recognition by the Ro autoantigen. Nat. Struct. Mol. Biol. 13: 1002-1009.

Gendron, M., Roberge, D., and Boire, G. 2001. Heterogeneity of human
Ro ribonucleoproteins (RNPS): Nuclear retention of Ro RNPS containing the human hY5 RNA in human and mouse cells. Clin. Exp. Immunol. 125: 162-168.

Green, C.D., Long, K.S., Shi, H., and Wolin, S.L. 1998. Binding of the 60-kDa Ro autoantigen to Y RNAs: Evidence for recognition in the major groove of a conserved helix. RNA 4: 750-765.

Hogg, J.R. and Collins, K. 2007. RNA-based affinity purification reveals 7SK RNPs with distinct composition and regulation. RNA 13: 868880.

Labbe, J.C., Hekimi, S., and Rokeach, L.A. 1999. The levels of the RoRNP-associated Y RNA are dependent upon the presence of ROP-1, the Caenorhabditis elegans Ro60 protein. Genetics 151: 143150.

Liu, J., He, L., Collins, I., Ge, H., Libutti, D., Li, J., Egly, J.M., and Levens, D. 2000. The FBP interacting repressor targets TFIIH to inhibit activated transcription. Mol. Cell 5: 331-341.

Maraia, R., Sakulich, A.L., Brinkmann, E., and Green, E.D. 1996. Gene encoding human Ro-associated autoantigen Y5 RNA. Nucleic Acids Res. 24: 3552-3559.

Niranjanakumari, S., Lasda, E., Brazas, R., and Garcia-Blanco, M.A. 2002. Reversible cross-linking combined with immunoprecipitation to study RNA-protein interactions in vivo. Methods 26: 182-190.

O'Brien, C.A. and Wolin, S.L. 1994. A possible role for the $60-\mathrm{kD}$ Ro autoantigen in a discard pathway for defective $5 \mathrm{~S}$ rRNA precursors. Genes \& Dev. 8: 2891-2903.

Page-McCaw, P.S., Amonlirdviman, K., and Sharp, P.A. 1999. PUF60: A novel U2AF65-related splicing activity. RNA 5: 1548-1560.

Perreault, J., Perreault, J.P., and Boire, G. 2007. The Ro associated Y RNAs in metazoans: Evolution and diversification. Mol. Biol. Evol. 24: $1678-1689$.

Puig, O., Caspary, F., Rigaut, G., Rutz, B., Bouveret, E., Bragado-Nilsson, E., Wilm, M., and Séraphin, B. 2001. The tandem affinity purification (TAP) method: A general procedure of protein complex purification. Methods 24: 218-229.

Shi, H., O'Brien, C.A., Van Horn, D.J., and Wolin, S.L. 1996. A misfolded form of $5 \mathrm{~S}$ rRNA is complexed with the Ro and La autoantigens. RNA 2: 769-784.

Stein, A.J., Fuchs, G., Fu, C., Wolin, S.L., and Reinisch, K.M. 2005. Structural insights into RNA quality control: The Ro autoantigen binds misfolded RNAs via its central cavity. Cell 121: 529-539.

Steitz, J.A., Berg, C., Hendrick, J.P., La Branche-Chabot, H., Metspalu, A., Rinke, J., and Yario, T. 1988. A 5S rRNA/L5 complex is a precursor to ribosome assembly in mammalian cells. J. Cell Biol. 106: 545-556.

Taft, R.J., Pheasant, M., and Mattick, J.S. 2007. The relationship between non-protein-coding DNA and eukaryotic complexity. Bioessays 29: 288-299. 


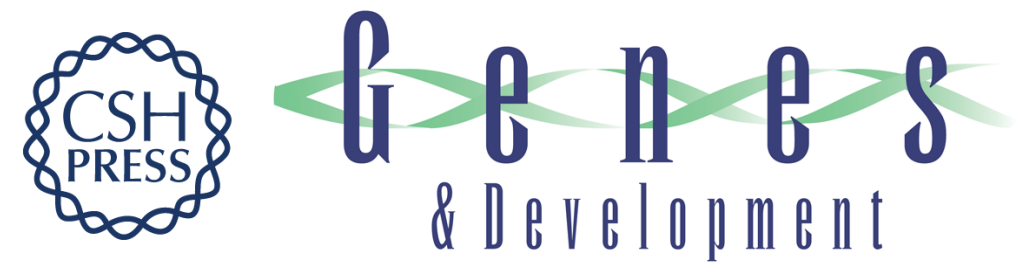

\title{
Human Y5 RNA specializes a Ro ribonucleoprotein for 5S ribosomal RNA quality control
}

\author{
J. Robert Hogg and Kathleen Collins
}

Genes Dev. 2007, 21:

Access the most recent version at doi:10.1101/gad.1603907

\section{Supplemental http://genesdev.cshlp.org/content/suppl/2007/11/15/21.23.3067.DC1 Material}

References This article cites 21 articles, 9 of which can be accessed free at: http://genesdev.cshlp.org/content/21/23/3067.full.html\#ref-list-1

\section{License}

Email Alerting

Receive free email alerts when new articles cite this article - sign up in the box at the top Service 